\title{
Grease flow in an elbow channel
}

\author{
Lars G. Westerberg • Josep \\ Farré-Lladós • Jinxia Li • Erik Höglund · \\ Jasmina Casals-Terré
}

Received: date / Accepted: date

\begin{abstract}
Lubricating greases are materials characterized by a complex nonNewtonian rheology. This study is a continuation of previous studies on the coupling between grease rheology and grease flow in a straight channel- and concentric cylinder geometry. The problem has been modeled and validated with experiments. Here the flow in an elbow channel introducing a non-symmetric distribution of shear stress throughout the cross section of the channel elbow, as well as varying shear rates through the transition from the channel inlet to the outlet. The flow has been modeled both for higher flow rates and for creep flow. The influence of the grease rheology and flow conditions to wall slip, shear banding and an observed stick-slip type of motion observed for low flow rates are presented. The effect on the flow of the applied pressure is also investigated showing that the flow is sensitive to the pressure in the angular $(\phi)$ direction of the elbow. For high flow rates it is shown that flow separation occur with a resulting circulation in the elbow.
\end{abstract}

Keywords Grease flow · micro Particle Image Velocimetry · Herschel-Bulkley rheology · Analytical modeling · Wall slip · Shear banding · Stick-slip flow · Flow separation

Lars G. Westerbere*

Division of Fluid and Experimental Mechanics, Luleå University of Technology, SE-971 87 Luleå, Sweden

*E-mail: lars-goran.westerberg@ltu.se

Josep Farré-Lladós · Jasmina Casals-Terré

Department of Mechanical Engineering, UPC - Technical University of Catalonia, Colom 7-11 08222 Terrassa (BCN), Spain.

Jinxia Li · Erik Höglund

Division of Machine Elements, Luleå University of Technology, SE-971 87 Luleå, Sweden 


\section{Introduction}

Grease and oil are widely used to lubricate moving mechanical parts in various mechanical systems such as gearboxes, engines and hub-units for trucks and passenger cars. Grease has in many applications been found to be preferable to oil as it due to its characteristic consistency prevent leakage and also contribute to a systems sealing properties. Grease also adheres to the surfaces which prevents corrosion and contribute to a lower friction. The main disadvantage with grease lubrication is the need of relubrication as the lifetime of the machine element often exceeds the lifetime of the grease.

Grease is a multi-phase material composed of a thickener, base oil and additives. The thickener determines the greases basic properties and forms a network structure in which the base oil is kept through Van der Waals- and capillary forces [4]. The thickener is also used to classify greases into different types [20], p.49. A typical distribution is usually $80-90 \%$ oil and 10-20\% thickener.

The thickener induce the grease's characteristic visco-elastic and yield behaviour [13], meaning the viscosity of the grease is strongly dependent on the shear rate (usually decreasing with increasing shear rate, so called shear thinning [20], p.112) and that the onset of flow is dependent on a threshold value of the shear stress. These properties gives grease different lubrication properties compared to oil. Oils have a Newtonian rheology which enables the running tracks in loaded contacts to be sufficiently replenished as the flow responds to the centrifugal forces and shear stresses induced by the motion of the geometry. This condition is necessary in order to have an optimum lubrication as the lubricant is pushed away from the contact due to the high load (shear stresses), which in turn implies a reduced amount of available lubricant in the running tracks. Greases however, will not without much difficulty flow back into the contact once they initially have been pushed out. Baart et al. [2] showed that the replenishment in the tracks of loaded contacts for grease lubrication also is supported by oil bleeding. However, as presented by Lugt et al. [21] oil bleeding may negatively affect the replenishment if the grease is in the wrong place. Also, too much grease will increase the friction due to heavy churning.

In order to understand and being able to optimize the lubrication mechanism, an understanding of grease flow dynamics is important. Further, a good lubrication is dependent on a sufficient supply of grease into the contacts. In lubrication systems the grease is pumped from a reservoir through pipes connected by means of elbow- and t-junction fittings. Here the grease flow dynamics in such curved geometries plays an important role for the pumpability of grease through these systems.

Studies of grease flow in pipes has been done by e.g. Mahncke and Tabor [22], and by Froishterer et al. [12]. Radulescu et al. [24] investigated the flow in pipes with discontinuities, which also could be applied to the flow in bearings. This work is a continuation of previous studies of the flow in closed straight channels $[29,17]$ and in a $3 \mathrm{D}$ double restriction seal geometry $[14,1$, 
18]. A channel with a $90^{\circ}$ elbow is used and an analytical model of the flow is developed and combined with experimental data from micro Particle Image Velocimetry $(\mu \mathrm{PIV})$ measurements of the flow in the elbow. This work is motivated by the value of an increases understanding of the flow motion of lubricating greases in curved geometries, both with respect to the fundamental scientific knowledge of greases fluid dynamics, but also to the engineering application of lubrication systems. The present study is also valuable as validation for numerical models.

\section{Analytical model of the flow in the channel elbow}

In this part an analytical model of the flow in the channel elbow is presented. Cylindrical coordinates $(r, \phi, z)$ are used to describe the flow; see Fig. 1 for a schematic view of the geometry. For the $\mu \mathrm{PIV}$ measurements presented in $\S 3$ a channel with a rectangular cross section of $250 \times 1000 \mu \mathrm{m}$ is used. The elbow section channel have an inner/outer radius of $0.875 / 1.125 \mathrm{~mm}$. For a corresponding model of the flow in the straight sections the reader is referred to the work by Westerberg et al. [29]. The flow is considered incompressible and the velocity $\mathbf{u}$ is considered to only have a component in the $\phi$-direction and only be dependent on the radial position, i.e. $\mathbf{u}=u_{\phi}(r)$. This means that the equation of motion solely can be treated to govern the flow. With one unknown velocity component the continuity equation is redundant. On tensor form the equation of motion reads

$$
\rho \frac{D u_{i}}{D t}=-p_{i}+\rho F_{i}+\tau_{i j, j},
$$

where $D / D t$ is the material derivative, $\rho$ the grease density, $u_{i}$ the velocity field, $\tau_{i j}$ the stress tensor, $p_{i}$ the pressure gradient, and $F_{i}$ a volume force which for the present case is gravity which in turn can be considered negligible. Considering the definition of the material derivative the equation of motion is written

$$
\rho \frac{\partial \mathbf{u}}{\partial t}+\rho(\mathbf{u} \cdot \nabla) \mathbf{u}=-\nabla p+\nabla \cdot \tau_{i j}
$$

Considering a stationary flow the time derivative of the velocity is zero. Further, considering a 1D flow the velocity in the elbow only has a component in the longitudinal $(\phi)$ direction, and the velocity is only dependent on the radial position in the elbow $\left(\mathbf{u}=u_{\phi}(r)\right)$. This means that the only non-zero component of Eq. (2) is the $\phi$-component. Consequently the only non-zero component of the shear stress tensor is $\tau_{r \phi}$ which equals $\tau_{\phi r}$ as the stress tensor is symmetric $([5], \S 3.2)$. Using the definition of the Nabla operator in cylindrical coordinates Eq. (2) then reduces to ([5], p. 612)

$$
0=\frac{1}{r^{2}} \frac{\partial}{\partial r}\left(r^{2} \tau_{r \phi}\right)-\frac{1}{r} \frac{\partial p}{\partial \phi} .
$$


For a Poiseuille flow in a straight channel the pressure distribution can be considered to decrease linearly along the length of the channel (i.e. in the main flow direction $(x)$ and vary in the transverse direction $(y)$ to the main flow direction such that

$$
p=\alpha x+f(y)
$$

where $\alpha$ is a negative constant with negative value and $f(y)$ is a general function whose expression not is known. The pressure gradient then reads

$$
-\frac{\partial p}{\partial x}=|\alpha|
$$

For the case of a flow in an elbow channel the pressure distribution can be considered analogous comparing the azimuthal cylindrical coordinate $\phi$ with $x$ in the straight channel. Curved channel flow is quite a large area within the fluid mechanical community as the curvature(s) easily for Newtonian fluids like water induce several interesting phenomena such as flow separation and instabilities. The work by Dean [8] is a classical paper within the field and for the flow in the elbow in the present analysis we consider an analogous pressure distribution

$$
p=\alpha \phi+f(r) .
$$

$\alpha$ is a constant and $f(r)$ an arbitrary function. A negative $\alpha$-value means that the pressure varies across a section of the elbow but decreases along the central surface of the channel similar to the flow in a straight channel. Eq. (6) then yields $\partial p / \partial \phi=\alpha$, and from Eq. (3) it follows that

$$
\frac{\partial}{\partial r}\left(r^{2} \tau_{r \phi}\right)=-r \alpha
$$

Solving for $\tau_{r \phi}$ results in (neglecting the indexes for simplicity)

$$
\tau=-\frac{\alpha}{2}+\frac{C_{1}}{r^{2}}
$$

where $C_{1}$ is a constant. The Herschel-Bulkley rheology model is considered to govern the relation between the shear forces and the shear rate in the grease. In cylindrical coordinates and for the present flow condition $\left(\mathbf{u}=u_{\phi}(r)\right)$ the Herschel-Bulkley model reads [18]

$$
\tau=\tau_{0}+K\left\{r \frac{\partial}{\partial r}\left(\frac{u_{\phi}}{r}\right)\right\}^{n}
$$


Here $\tau_{0}$ is the yield stress, $K$ the consistency of the grease, and $n$ the power law exponent which for a shear thinning material like grease typically is less than one. Eq. (9) into Eq. (8) gives

$$
K\left\{r \frac{\partial}{\partial r}\left(\frac{u_{\phi}}{r}\right)\right\}^{n}+\tau_{0}=-\frac{\alpha}{2}+\frac{C_{1}}{r^{2}},
$$

i.e.

$$
r \frac{\partial}{\partial r}\left(\frac{u_{\phi}}{r}\right)=\frac{1}{K^{\frac{1}{n}}}\left(-\frac{\alpha}{2}+\frac{C_{1}}{r^{2}}-\tau_{0}\right)^{\frac{1}{n}}
$$

Solving for $u_{\phi}$ results in

$$
u_{\phi}(r)=\frac{r}{K^{\frac{1}{n}}} \int \frac{1}{r}\left(-\frac{\alpha}{2}+\frac{C_{1}}{r^{2}}-\tau_{0}\right)^{\frac{1}{n}} d r+C_{2} r,
$$

which is a general solution for the velocity in the viscous region in connection to respective boundary in the channel elbow; see Fig. 2. $C_{2}$ is a new constant. The velocity profile has in the presence of wall slip three characteristic regions in connection to each boundary, going from respective boundary towards the middle of the elbow [29]: a very thin slip layer, a viscous region where the grease is fully yielded, i.e. $|\tau|>\tau_{0}$, and a plug region where the grease behaves as a solid body $|\tau|<\tau_{0}$. For the case of no slip and no plug flow the two viscous layers fill the channel width. The slip layer is identified from the experiments by extrapolating the velocity profile towards the origin representing the inner radius of the channel, and towards opposite boundary (outer radius); cf. Fig. 1. For a no slip condition the velocity would approach zero continuously, but as illustrated in the figure this is not the case; rather than having a continuous behavior of the velocity profile, the velocity gradient appears to be significantly higher in the region between the solid boundary (inner radius) and the first registered data point for the velocity. For a flow scenario as in Fig. 2 the slip effect is greater close to the inner radius compared to the corresponding effect at the opposite boundary.

For a flow in a straight channel, the velocity profile is symmetric along the centerline of the channel; this has not to be the case in a curved channel as the non-symmetric geometry may induce an increasing velocity in the positive radial direction and hence induce asymmetry in the flow. The yield point is the location(s) in the flow where the shear stress in the grease equals the yield stress. For the case of a non-existing plug layer, the yield point then is the point of maximum velocity; cf. a Newtonian Poiseuille flow in a straight channel which has a characteristic parabolical velocity profile. The yield point in this case is at the channel center. For the case of a plug flow in a non-symmetric flow as in the channel elbow, the velocity profile has two yield points: one on each side of the centerline of the channel. These points constitute the limits of 
the plug region. The analytical velocity profile across the channel elbow is then built by the plug region (if present), and the viscous solutions in connection to the plug region on respective side of the channel centerline; see Fig. 2.

Matching the velocity profile with the $\mu \mathrm{PIV}$ measurements, the data points of the velocity can be used as boundary conditions to solve for the unknown constants in Eq. (12). In channel flows a no slip condition at the channel wall is typically used. However, if slip is present the no slip condition is no longer valid. Following the methodology presented in Westerberg et al. [29] the velocity data point being located closest to the channel wall is considered as boundary condition in this paper ( $u_{1}$ in Fig. 2$)$. Eq. (12) can then be re-written as

$$
u_{\phi}(r)=u_{\phi 1}(r)=\frac{r}{K^{\frac{1}{n}}} \int_{r_{1}}^{r} \frac{1}{r}\left(-\frac{\alpha}{2}+\frac{C_{1}}{r^{2}}-\tau_{0}\right)^{\frac{1}{n}} d r+r \frac{u_{1}}{r_{1}} .
$$

A general solution for an arbitrary value of $n$ does not exist but for certain fractions a solution can be found. Two fractions are $n=1 / 2$ and $n=1 / 3$ respectively. The greases used in this paper all have an $n$-value of the order of 1; see Tab. 1. In the studies by Li et. al [18] and Westerberg et. al [29] it is also shown that $K$ and $\tau_{0}$ have larger impact on the flow as they span over larger magnitudes. It is found that a good match between the analytical model and the measured velocity profile is obtained for $n=1 / 2$ for the case of a reduced pressure and yield stress value. This behavior is likely due to the changed rheology of the grease with a reduced $n$-value causing the applied 1D assumption to break down. The $n$-value is henceforth considered to be 1 . For $n=1$ the integral in Eq. (13) is straightforward to calculate. The solution for $u_{\phi}$ then reads

$$
u_{\phi}(r)=\frac{r}{K}\left[\left(\tau_{0}+\alpha\right) \ln \left(\frac{r_{1}}{r}\right)+\frac{C_{1}}{2}\left(\frac{1}{r_{1}^{2}}-\frac{1}{r^{2}}\right)\right]+\frac{r u_{1}}{r_{1}} .
$$

To solve for $C_{1}$ the yield point in the measured velocity profile is identified. With $r_{p 1}$ as the location of the yield point and the corresponding velocity $u_{p 1}$ the solution for $C_{1}$ yields

$$
C_{1}=-\frac{r_{1} r_{p 1}}{r_{1}^{2}-r_{p 1}^{2}}\left[r_{p 1} r_{1}\left(2 \tau_{0}+\alpha\right) \ln \left(\frac{r_{p 1}}{r_{1}}\right)+2 K\left(u_{p 1} r_{1}-r_{p 1} u_{1}\right)\right] .
$$

The solution for the velocity profile presented above constitute $u_{\phi 1}(r)$ in Fig. 2 . The solution for $u_{\phi 2}$ is obtained analogously considering the first measured velocity calculated from the outer boundary, located at $r=r_{2}$, and the velocity at the yield point $\left(r=r_{p 2}\right)$. Important here is that the $r$-coordinate in Eq. (14) is defined positive in the normal direction to the channel boundary pointing into the channel. 


\section{Grease flow measurements using $\mu$ PIV}

In this section results from the flow visualizations are presented and discussed. The $\mu \mathrm{PIV}$ measurements have been conducted in a micro-channel featuring a 90 degree elbow; see Fig. 3 for a view of the channel setup. The channel has a rectangular cross section of $250 \times 1000 \mu \mathrm{m}$. The radius at the channel centerline is $1 \mathrm{~mm}$. The channel is manufactured using a $3 \mathrm{D}$ printer from Stratasys, model Objet 30. The channel is built in the polymeric material VeroWhitePlus RGD835, and UV curing glue is used to seal the joint of the micro-channel part and the glass. Fig. $3 \mathrm{~b}$ shows the $3 \mathrm{D}$ model of the RPT part and Fig. 4 the steps followed to manufacture the micro-channel. More details about the $3 \mathrm{D}$ printing operation to build the present channels can be found in the paper by Farré et al. [11]. Fig. 1 shows a schematic drawing of the micro-channel and the locations where the $\mu \mathrm{PIV}$ measurements have been performed in the straight section and the elbow. The exact position within the dashed regions cannot be defined as no reference point can be introduced in the micro-channels. However, the measurements are not performed any closer than $5 \mathrm{~mm}$ from the inlet in the straight section, meaning the flow is fully developed considering the Hagen-Poiseuille law [9].

The $\mu \mathrm{PIV}$ experimental setup is shown in Fig. 5a and the principles behind $\mathrm{PIV}$ is presented in Fig. 5b. The experimental setup consists of a syringe to inject the grease into the channel, a syringe pump to control the flow rate, a laser to illuminate the flow, a microscope with the corresponding objective(s) and a high speed camera to capture the flow motion, and a computer with PIV software to calculate the velocity of the flow. The main principle of $\mu \mathrm{PIV}$ is to take a series of double frame images of the particle seeded grease and to analyze the images in the computer to visualize the flow motion. Here the tracer particles used are dry-powder fluorescent MF Rhodamine B particles from Microparticles GmbH (www.microparticles.de) having a diameter of $3.23 \pm 0.06 \mu \mathrm{m}$. The microscope objective has a 20x magnification. The images within each double frame image-pair are separated by a small time step enabling the motion of the tracer particles to be calculated using a correlation routine. Each experiment in the present study comprises the average of a total of 500 image pairs. The system used for these experiments is a commercial system by Dantec A/S which includes the software Dynamic Studio in which the PIV routine is incorporated. The method of PIV is widely used to visualize fluid flow, it is not limited to small scale geometries; it can theoretically be applied to any scale as long as it is possible to record the motion of the flow. The main difference between $\mu \mathrm{PIV}$ and "macro" PIV is that in $\mu \mathrm{PIV}$ the entire flow volume is illuminated by the laser light and the actual measuring plane is set by the focal depth of the microscope, while for macro PIV a laser sheet - comprising the measuring plane - is formed using imaging optics; cf. Fig. 5b. For more details on PIV the book by Raffel et al. [25] is recommended. 


\section{Results and discussion}

In this section velocity profiles from flow visualizations are presented for the grease flow in the inlet (straight section upstream of the elbow), elbow and outlet respectively; cf. Fig. 1. The velocity profiles at the elbow have been compared to the analytical model. Three different lithium greases with NLGI grade 00, 1 and 2 have been used; see Tab. 1 for rheology data of the actual greases based on the Herschel-Bukley rheology model. In order to obtain best possible fit the values of the grease consistency $K$ and yield stress $\tau_{0}$, and the angular pressure parameter $\alpha$ have been adapted. The influence of the angular pressure in the elbow have also been analyzed separately as it was found that the $\alpha$-value heavily determines the characteristics of the flow in the elbow. Of specific interest are also wall slip effects, shear banding, and the transition of the flow as the grease moves through the elbow. These aspects are analyzed in separate subsections below.

A justified question is how correct it is to consider the rheological parameters as free parameters for the curve fit as we have a measured value obtained from a rheometer. Imprtant here is that rheometer measurements not are without error sources; there are in fact many error sources related to these measurements, see e.g. $\$ 5.2 .2$ in the book by Lugt [20]. Wall slip is for instance not considered in rheometers and the grease is assumed to be uniformly sheared throughout the gap. A couple of papers by the authors have shown that these conditions in fact are violated for the case of grease flow [29,18]. A consequence of this is the question how reliable the values from a rheometer are in terms of grease rheology. It is hence of interest to investigate eventual discrepancies between the rheometer values and values obtained from the fit between the analytical model and the measured velocity profiles.

The flow rates considered in this paper range from $0.002 \mathrm{ml} / \mathrm{min}$ to $0.5 \mathrm{ml} / \mathrm{min}$ which are the limits of the syringe pump. Higher (or lower) flow rates may be of interest but this range is considered satisfactory at present stage. The experimental results have of course a specific value on their own, but in the context of a combined analytical model with experimental data, the latter has an important role as validation for the analytical model and for future numerical models.

The values of the rheological parameters in the Herschel-Bulkley model have indeed been measured for each grease as mentioned above. Of interest is to compare these values with the values obtained from the curve fit between the analytical model and the $\mu \mathrm{PIV}$ data. Rheometer measurements are typically affected by errors as Lugt reports [20], chapter 5.2.2. For the low- to mid Reynolds number flow covered by the measurements, the analytical model is considered robust. For increasing Reynolds numbers the flow will eventually become turbulent and then the model is no longer valid. By comparing the matched values of the rheology model constants with the ones obtained from the rheometer measurements we can get an indication on the reliability of the measured values. 
4.1 Flow separation in the elbow: influence of the driving pressure ( $\alpha$-value) on the flow

Fig. 6 shows the behavior of the velocity across the elbow as $\alpha$ grows in magnitude. As $\alpha$ increases the trend is that the flow approaches a flow reversal in connection to the boundaries of the elbow. Here 'reversal' means that the flow in connection to the curved boundaries in the elbow change direction and hence induce an increased shear in the flow, which in turn lead to a vortical flow (circulation) in the elbow. Within the pure scientific context the mechanisms behind the reversal are of interest to build a detailed understanding of the physics of the grease flow. Flow reversal is characterized by the negative velocity which start to appear for $\alpha=-500$ and which is fully present for $\alpha=-1000$. Flow reversal is typically present in connection to flow separation initialized by an adverse pressure gradient caused by the curved geometry [15]. Flow separation means that the flow undergoes a transition from being detached to the channel boundary to inherit a vortical structure. It is of interest to investigate what happens as the flow rate increases from the actual maximum value of $0.5 \mathrm{ml} / \mathrm{min}$, and when flow separation is initiated. As introduce above, this will however require a new test rig which can handle required pressure drops to induce higher velocities in the flow, and is left for a future development of the present study. The working hypothesis is though that flow separation is responsible for the observed flow reversal in the elbow. It should be noted that there is a great difference between laminar separation and turbulent separation; the latter occurring at (very) high Reynolds numbers (see below) which should be considered exotic when it comes to grease flow due to the high viscosity of the grease. In the limit of infinite shear rate when the viscosity of the grease approaches the base oil viscosity turbulence may be present in the flow if the velocity is high enough, but for the cases presented in the present paper the flow is laminar. The core in the working hypothesis is a combination of the elbow geometry which comprise two sharp curvatures, and the onset of the flow reversal as $\alpha$ increases. Also, the flow reversal effect is slightly more accentuated in connection to the inner boundary of the elbow which has a smaller curvature radius than the outer radius hence causing a stronger adverse pressure gradient and consequently a more pronounced separation. Generally, in order to avoid separation, the boundary geometry should be varied gradually in order to follow the flow streamlines; this is however not the case in the elbow as the flow experiences a fast transition from an infinite curvature radius (i.e. a straight channel flow) to a curvature of $1 \mathrm{~mm}$. Another interesting observation is that the critical $\alpha$-value $\left(\alpha_{\mathrm{c}}\right)$ for when the flow reversal is initiated is highly dependent on the NLGI grade of the grease. For NLGI $00 \alpha_{\mathrm{c}} \approx-100$ while for NLGI $2 \alpha_{\mathrm{c}} \approx-1000$ to be compared for the corresponding value of -500 for the NLGI 1-grease. This observation makes sense in terms of flow separation as a less viscous fluid will have a larger Reynolds number compared to a more viscous fluid for the same velocity. The (dimensionless) Reynolds number is the ratio between the inertial- and viscous forces in the flow according to 


$$
R_{e}=\frac{L U}{\eta},
$$

where $L$ is a characteristic length scale (in channel flow typically the channel width), $U$ a characteristic velocity (e.g. the mean velocity in the channel or the maximum velocity), and $\eta$ the kinematic viscosity which in turn is dependent on the shear rate as lubricating greases are shear thinning materials. The location where flow separation occur is not dependent on the Reynolds number [15], but the overall flow characteristics - including the presence of separation or not - is heavily influenced by the Reynolds number. The impact of the curvature of the boundaries is also shown when comparing Fig. 6(a)-(b): in subfigure (a) the analytical model does not fully match the measured velocity close to the inner boundary $\left(0<r^{*}<0.3\right)$, but as the pressure is increased in (b) the match is better. This indicates that the pressure decrease with $r^{*}$.

\subsection{Shear banding}

Shear banding is a phenomena in the flow induced by instabilities in the grease phases (base oil and thickener), causing the shear rate in the flow to vary across the channel width $[10,16]$. One important application where the occurrence of shear banding have a direct impact on the results is in rheometers. Here a uniform shear rate is assumed to be present in the gap when measuring the torque needed to rotate the plate or cylinder - depending on actual rheometer type. With shear banding present in the flow, the conditions considered for the measurements are obviously violated inducing errors in the rheology measurements. When modeling the flow shear banding is not normally accounted for. In the analytical model presented in this paper the shear rate is assumed to be homogeneously distributed across the channel; in the plotted velocity profiles in Figs. 6-7 this is showed by the continuously varying velocity in the viscous layer between the channel wall and the position of the plug region if present, otherwise the velocity peak in the channel. Shear banding may be responsible for the deviation between the analytical model and the $\mu$ PIV measurements viewed in Fig. 7. It is close at hand to expect that the pressure can cause the deviation with respect to what is discussed in the previous subsection, but the pressure affect the velocity continuously from the location of the plug region as viewed in Fig. 6. From Fig. 7a it follows that in the viscous layer in connection to the inner radius (located approximately in the region $0<r^{*}<0.4$ ) the general trend is a parabolical velocity profile as described by the analytical solution. The deviation from the flow represented by the velocity $u^{*} \approx 0.3$ at $r^{*} \approx 0.15$ indicates a local effect. A similar behavior is seen for the higher flow rate case in Fig. 7c. Here the velocity profiles in connection to the outer radius show a more continuous concave shape; this effect is coupled to the geometry where the concave outer wall induce a more continuous flow compared to the convex inner radius which resembles the geometry of a rounded backward facing step. Lerouge at al. [16] (Fig. III.1, p.19) show that shear banding due to 
the discontinuous shear rate distribution in the flow is characterized by notches in the graph when plotting the flow velocity gradient versus the velocity. The notches are the locations of the critical shear rates in the flow. In a shear stress/shear rate plot the shear banding effect is viewed as an unstable region within the limits formed by the critical shear rates. With this background it can be argued that the location of the critical shear rates in Fig. 7a are at $r^{*} \approx 0.15$ and $r^{*} \approx 0.25$, and $r^{*} \approx 0.8$ and $r^{*} \approx 0.9$ at respective viscous layers. No general conclusion can be drawn regarding the shear banding effect related to the NLGI grade of the grease. In terms of the flow rate the tendency is that the effect we refer to as shear banding in this section, is reduced for higher flow rates. This result can be related to the origin of shear banding illustrated by Lerouge et al. which can be applicable to greases considering a similarity between the grease thickener and giant micelles: For high shear rates the thickener fibres are aligned with the flow while as the shear rate decreases the fibre orientation becomes more random causing a varying rheology in the grease. Consequently, if the flow rate is high enough the shear rate in the flow is high enough to form aligned fibres throughout the whole flow domain and hence the shear banding effect will vanish.

\subsection{Wall slip and flow evolution in the elbow channel}

Modeling channel flow, or flow in the gap in a rheometer, a no slip boundary condition is typically applied. In grease flow this condition is showed to easily be not fulfilled $[29,7,6]$. The origins of wall slip are not entirely understood; that phase separation is responsible for the slip in connection to the solid boundary most researchers agree on. Wall slip effects through a thin layer at the walls consisting of a less viscous (i.e. lower consistency index) grease than the bulk grease [6,23]. The lower consistency is by Barnes [3] suggested to be due to oil separation between the base oil and the grease thickener agent. The concept of wall slip can be divided into two categories: i) true slip, where there is a discontinuity in the velocity field at the fluid-solid interface, and ii) apparent slip, where there is an inhomogeneous thin layer of a fluid adjacent to the wall with different rheological properties to the bulk of fluid enabling fluid movement [27]. Li et al. [19] also showed that in a free-surface flow oil bleeding is present where the grease is sheared. This result supports the idea of oil bleeding being responsible for wall slip as oil bleeding in connection to the walls in closed (channel) flows in turn is supported by the high shear stresses close to the solid boundaries.

Fig. 7 shows that the wall slip effect is small in the elbow, both for the soft NLGI 00-grease and the thick NLGI 2-grease. The wall slip effect in the flow is characterized by $u^{*}=u_{\mathrm{slip}}^{*}>0$ when the velocity profile is extrapolated towards $r^{*}=0$. The resolution of the flow is finite and set by the microscope objective magnification, meaning the first measured velocity is somewhere between the solid wall and the distance set by the length between the data points in the velocity grid. As presented in $\S 3$ the micro-channel is built of a polymeric 
material (VeroWhitePlus). The channel surface roughness has an Ra value of $1.85 \mu \mathrm{m}$ with standard deviation of $0.78 \mu \mathrm{m}$ which should be compared to the channel Ra value in the work by Westerberg et al. [29] lying in the range 0.1-5 $\mu \mathrm{m}$. In that study it was concluded that these Ra values did not have any influence on the wall slip observed in the straight channel used. Czarny [7] on the other hand showed that the surface roughness has impact on the wall slip, but this yields for the case of a surface roughness of several orders of magnitude larger than considered by Westerberg et al who used bearing steels as reference. Westerberg et al. also showed that the slip effect is not dependent on the actual surface material which in their study comprised steel (AISI 52100), polyamide and brass. Another conclusion was that the slip decreases with increasing flow rate. For elbow channel flow the shear stress is higher in the elbow than in the straight sections (inlet and outlet) as the curvature of the geometry is included in the expression for the shear stress; see Eq. 9. Considering a constant velocity in the $\phi$-direction the shear stress is proportional to the inverse of the radial position $r$. Having negligible wall slip in the elbow section can hence be connected to the conclusion of a reduced wall slip with increasing shear forces. Czarny [7] also showed that wall slip was accentuated for low shear rates. In Fig. 8 velocity profiles at the inlet, elbow and outlet are compared. Considering subfigure (a) showing the low flow rate case for the NLGI 00-grease; if the velocity profile for the inlet and outlet regions in the channel (square and cross labeled curves) are extrapolated towards the inner- and outer boundary respectively a slip velocity of approximately 0.3 is obtained, while the corresponding slip velocity at the elbow (dotted curve) indicates a zero slip velocity. In (c) we see that that for a high flow rate the velocity profiles at all three locations have the same parabolical form. For the thick NLGI 2-grease ((b) and (d)) the difference in slip behavior is not as distinct; a clear observation is though that the length of the plug region is reduced at the elbow. The results by Westerberg et al. [29] also support this since they showed that the plug region decreases when the pressure drop (flow rate) is increased. An interesting observation when comparing the low- and high flow rates for the NLGI 2 grease is that the velocity profile in the elbow not is affected by the higher flow rate, indicating that the shear rate in the elbow even at low flow rates induces a fully yielded grease. These results agree with the conclusions made by Tamayol et al. [28] stating that the slip increases with the Knudsen number $(K n=\lambda / L$ i.e. [mean free path]/[characteristic length scale]): with an increasing consistency (which we can treat as a viscosity for the non-Newtonian grease) the mean free path increases and consequently $K n$ increases [26].

\subsection{Stick-slip type of motion during transitory period}

Another interesting observation when comparing the velocity profiles during increased flow rates was a variation in the maximum speed in the channel for a constant feeding flow rate. This behavior is strange considering the grease 
being a continuum flowing medium; with the same flow rate and constant channel cross section the maximum speed of the flow should remain constant. Based on these observations, the evolution of the maximum speed at the inlet during the transitory period has been investigated; see Fig. 9. Starting from rest the specified feeding flow rate is applied until stationary conditions are reached. A high- and low flow rate are considered. Comparing subfigure a and $\mathrm{b}$ it follows that stationary conditions are reached fast for the high flow rate case, while stationary conditions for the low flow rate are never reached; the speed shows a great variation as the pressure is built up in the system with a behavior similar to a stick-slip motion. An explanation of this result can be coupled to the non-homogeneous properties of the grease also shown as shear bands in the flow. As the pressure is built up in the grease the shear forces in the grease are spatially varying due to a varying composition of thickener and base oil which in turn induces varying rheological properties of the grease. This also means that the pressure distribution in the grease is varying. Once the shear stress (locally) in the grease exceeds the yield stress, the flow is released and the locally accumulated pressure introduces a high pressure gradient compared to the main driving pressure induced by the syringe pump, causing the speed of the grease flow to accelerate - which in Fig. 9a is shown as the rapid increases (peaks) in the measured speed. Once this transient effect is decaying the shear stress in the grease will approach the yield stress once again causing a retardation of the motion of the grease. This effect is visualized by the sudden drop in speed shown in Fig. 9a. For the case of a high flow rate the shear forces in the grease exceeds the yield stress throughout the whole domain, causing a continuous deformation of the grease and a continuous flow in the channel. The coupling of the observed phenomena to the yield stress behavior of the grease is observed when comparing the behavior of the NLGI 00- and NLGI 2-grease: for the NLGI 2-grease flow at high flow rates the transition from the onset of grease motion to stationary flow occur on a short time scale, while the NLGI 00-grease due to its negligible yield stress start to move when the feeding pressure is set and hence the corresponding transition time from the onset of grease motion to a stationary speed is longer. The time needed to achieve a stationary speed calculated from the onset of the driving pressure is however equal for both greases. The measured stick-slip type of behavior is interesting on a fundamental level with respect to the grease rheology and its coupling to the grease flow, but also with respect to applications such as pumping/feeding of grease - e.g. in lubrication systems discussed in $\S 4.2$. With a too low feeding pressure the flow rate of grease into the actual component will vary significantly, which in turn may have negative consequences on the desired lubrication due to too much grease or practically no inflow of grease. This scenario becomes accentuated at low temperatures as the grease viscosity and yield stress change dramatically. 
4.5 Grease flow in pipes used in lubrication systems

The observed flow characteristics presented above present in elbow geometries, may be of certain interest for lubrication systems. The present work does not cover research on lubrication systems as such, but still the results may have value for this engineering application as elbow- and t-joint fittings are occurring in these systems. Problems with disruptions in the feeding of grease through the pipes and fittings are not uncommon (Victoria Van Camp, Vice President Product Management and R\&D at SKF Lubrication Systems, St Louis, US. Private communication May 23, 2014). Lubrication systems have an important role in lubrication as the right amount of grease is supposed to be delivered to the right location at the right time. A challenge is to find a grease whose properties satisfies both the lubrications systems'- and the bearing's requirements. And here knowledge of the flow behaviour observed in this paper is of interest.

From the model observations it follows that the circulation in the elbow due to flow separation increases with the driving pressure. The condition for when circulation in the elbow is initiated is also strongly dependent on the consistency index ( $K$ in the Herschel-Bulkley rheology model) and on the shear thinning parameter $n(<1)$. This result seems natural as a stiffer grease due to lower bulk inertial forces and higher viscosity inherits a resistance to separate from the elbow walls. For a decreasing $n$-value the viscosity of the grease will be lower for the same shear rate, meaning that circulation in the elbow theoretically could be initiated, or ceased, for constant pressure by changing the shear thinning properties of the grease: increase the $n$-value to prevent circulation, or decrease the $n$-value to initiate circulation. The circulation in the elbow is initiated by the shear in the flow induced by the curved elbow geometry; the flow in the straight section(s) does however not experience the same magnitude of shear stresses meaning the well-ordered plug-, or parabolical upstream of the elbow enter a region with a swirling flow. This swirl is likely to act as an obstacle for the approaching flow, or in worst case block the entire flow. Other effects such as phase separation (oil bleeding) due to the shear stresses in the elbow may contribute to block the elbow fitting. So in practical terms, based on these results blocked pipes could be avoided by decreasing the feeding pressure, increase the curvature radius of the fitting parts of the pipes, and/or have a grease with less pronounced shear thinning properties.

The stick-slip type of motion observed when investigating the speed in the channel (§4.4) also is interesting for the flow in lubrication systems. For low feeding flow rates where the shear stress in the bulk grease balances on the yield stress value, the pressure is locally increased which in turn increases the shear stress until the yield condition is passed and a rapid increase in velocity is obtained. With the released flow the shear stress magnitude distribution in the flow decreases, implying the grease will approach the yield threshold condition once again. These variations in the flow will contribute to challenges in terms of keeping a uniform flow rate into the system where the lubricated 
contact surfaces are located. The pumpability of grease through the pipes in lubrication systems will also be strongly affected by low temperatures as the grease stop bleeding oil and its flow properties will be different due to a stiffer (i.e. more viscous) grease [20], p.19.

Wall slip is an interesting phenomena: as presented in $\S 4.3$ there is no consensus on whether the slip is caused by a large concentration gradient of base oil or thickener. If we as a working hypothesis consider the former to be the primary reason for the existence of wall slip in grease flow, it is not unlikely that wall slip may be of value for the pumpability as slip reduces the friction and consequently a lower pressure is needed.

\section{Summary and concluding remarks}

In this paper the flow of lubricating greases have been analyzed in an elbow channel with a rectangular cross section of $250 \times 1000 \mu \mathrm{m}$. The study comprise a dual experimental/analytical approach where the method of micro Particle Image Velocimetry $(\mu \mathrm{PIV})$ is used to measure and visualize the flow in the channel. The analytical model is built from the fluid equation of motion considering the Herschel-Bulkley rheology model. Three Lithium-based greases with NLGI grade 00, 1 and 2 respectively have been used and the flow has been analyzed for flow rates in the range $0.002 \mathrm{ml} / \mathrm{min}$ to $0.5 \mathrm{ml} / \mathrm{min}$. It is shown that there is a good match between the analytical model of the flow in the elbow and the measured velocity profiles. The analytical solution for the velocity across the elbow section is shown to be sensitive to the pressure in the angular direction ( $\phi$, considering cylindrical coordinates). As the pressure increases the velocity profile gradually approaches a reversal indicating that flow separation occurs in the elbow in connection to the solid boundaries. This result has a direct application in lubrication systems where an effective feed of grease from the reservoir to the component to be lubricated is highly important. With separation present in the flow, the possibility to effectively pump the grease through the pipe system is disturbed. Another finding with direct relevance to lubrication systems is that for low flow rates the grease experiences large velocity fluctuations in the channel, i.e. a stick-slip type of motion. This effect disappears for high flow rates, concluding that in order to achieve an even flow rate through (for instance) a lubrication system the applied flow rate itself cannot be too low. Flow visualizations show that wall slip is present and it is found to be reduced in the elbow compared to the inand outlet sections of the channel. The conclusion is that this effect - which mainly is observed for low flow rates and for the thinnest grease with NLGI grade 00 , is caused by the higher shear magnitude in the elbow flow caused by the curvature. For high flow rates the slip effect decreases and the overall flow is unaffected by the elbow. Shear banding due to a non-uniform distribution of the shear rate in the channel is also observed. 
Acknowledgements The authors would like to thank Dr. Torbjörn Green and Dr. Henrik Lycksam at the Division of Fluid and Experimental Mechanics, LTU for their help with the $\mu$ PIV measurements. This project has been funded by the Swedish Research Council (VR) and by KIC InnoEnergy.

\section{References}

1. Baart, P., Green, T.M., Li, J., Lundström, T.S., Westerberg, L.G., Höglund, E., Lugt, P.M.: The influence of speed, grease type, and temperature on radial contaminant particle migration in a double restriction seal. Trib. Trans. 54(6), 867-877 (2011). DOI:10.1080/10402004.2011.609308

2. Baart, P., van der Vorst, B., Lugt, P.M., van Ostayen, R.A.J.: Oil-bleeding model for lubricating grease based on viscous flow through a porous microstructure. Trib. Trans 53(3), 340-348 (2010). DOI: 10.1080/10402000903283326

3. Barnes, H.A., Walters, K.: The yield stress myth. Rheol. Acta 24, 323-326 (1985)

4. Bauer, W.H., Finkelstein, A.P., Wiberly, S.E.: Flow properties of lithium stearate-oil model greases as a function of soap concentration and temperature. ASLE Transactions 3(2), 215-224 (1960)

5. Bird, R.B., Armstrong, R.C., Hassager, O.: Dynamics of Polymeric Liquids, vol. 1 Fluid Mechanics. John Wiley \& Sons (1987). ISBN 0-471-80245-X

6. Bramhall, A.D., Hutton, J.F.: Wall effect in the flow of lubricating grease in plunger viscosimeters. Br. J. Appl. Phys. 11, 363-369 (1960)

7. Czarny, R.: The influence of surface material and topography on the wall effect of grease. Lubrication Science 14(2), 255-273 (2004)

8. Dean, W.R.: Fluid motion in a curved channel. Proc. R. Soc. Lond. A 121, 402-420 (1928). DOI: 10.1098/rspa.1928.0205

9. Delgado, M.A., Franco, J.M., Partal, P., Gallegos, C.: Experimental study of grease flow in pipelines. wall slip and air entrainment effects. Chemical Engineering and Processing 44, 805-817 (2005)

10. Dimitriou, C.J., Casanellas, L., Ober, T.J., McKinley, G.H.: Rheo-piv of a shear-banding wormlike micellar solution under large amplitude oscillatory shear. Rheologica Acta 51(5) (2012). DOI: 10.1007/s00397-012-0619-9

11. Farré-Lladós, J., Casals-Terré, J., Voltas, J., Westerberg, L.G.: The use of rapid prototyping techniques (rpt) to manufacture micro channels suitable for high operation pressures and $\mu$ piv. Rapid Prototyping Journal (2014). Submitted

12. Froishteter, G.B., Smorodinskii, E.L., Ishchuck, Y.L., Yurtin, L.O., Radionova, N.V. Calculation of hydraulic resistance in transport of lubricating greases through pipelines. Chemistry and Technology of Fuels and Oils 12(2), 129-133 (1976)

13. Gow, G.M.: The cey to grease rheology. Transactions of Mechanical Engineering, The Institution of Engineers, Australia, Special Issue Tribology ME16(3), 202-205 (1991)

14. Green, T.M., Baart, P., Westerberg, L.G., Lundström, T.S., Höglund, E., Lugt, P.M., Li, J.: A new method to visualize grease flow in a double restriction seal using micro particle image velocimetry. Trib. Trans. 54(5), 784-792 (2011). DOI: 10.1080/10402004.2011.604759

15. Kundu, P.K.: Fluid mechanics. Academic Press, Inc., San Diego, California, USA (1990) ISBN: 0-12-428770-0, Ch. 10.6-10.8.

16. Lerouge, S., Berret, J.F.: Shear-induced transitions and instabilities in surfactant wormlike micelles. Advances in Polym. Sci. 230, 1-71 (2010). DOI: 10.1007/978-3-642-135323

17. Li, J., Höglund, E., Westerberg, L.G., Green, T.M., Lundström, T.S., Lugt, P.M., Baart, P.: $\mu$ piv measurement of grease velocity profiles in channels with two different types of flow restrictions. Trib. Int. (2012). DOI: 10.1016/j.triboint.2012.03.00

18. Li, J., Westerberg, L.G., Höglund, E., Lugt, P.M., Baart, P.: Lubricating grease shear flow and boundary layers in a concentric cylinder configuration. Trib. Trans. 57(6) (2014). DOI: 10.1080/10402004.2014.937886 
19. Li, J., Westerberg, L.G., Höglund, E., Lugt, P.M., Baart, P.: Lubricating grease shear flow and boundary layers in a concentric cylinder configuration. In: 16th Nordic Symposium on Tribology. Danish Technological Institute (2014)

20. Lugt, P.M.: Grease Lubrication in Rolling Bearings. Wiley Tribology Series (2013). Print ISBN:9781118353912, Online ISBN: 9781118483961, DOI: 10.1002/9781118483961

21. Lugt, P.M., Velickov, S., Tripp, J.H.: On the chaotic behaviour of grease lubrication in rolling bearings. Trib. Trans. 52(5), 581-590 (2009). DOI: 10.1080/10402000902825713

22. Mahncke, H.E., Tabor, W.: A simple demonstration of flow type in greases. Lubr. Eng. 122(Jan-Feb), 22-28 (1955)

23. Pérez-González, J., López-Durán, J.J., Marín-Santibáñez, B.M., Rodríguez-González, F.: Rheo-piv of a yield-stress fluid in a capillary with slip at the wall. Rheol Acta $\mathbf{5 1}$ 937-946 (2012)

24. Radulescu, A.V., Bonneau, D., Hajjam, M.: A theoretical study of two-dimensional grease flow in regions with discontinuities. Lubrication Science 15(2), 163-171 (2003). DOI: $10.1002 /$ ls.3010150206

25. Raffel, M., Willert, C.E., Wereley, S.T., Kopenhans, J.: Particle Image Velocimetry. Springer, Berlin Heidelberg (2007). ISBN (print) 978-3-540-72307-3, ISBN (online) 9783-540-72308-0

26. Salmon, R.L.: Lectures on geophysical fluid dynamics, Ch. 1.11. Oxford University Press (1998). ISBN 0-19-510808-6

27. Sochi, T.: Slip at fluid-solid interface. Polym. Rev. 51 (2011). DOI: 10.1080/15583724.2011.615961

28. Tamayol, A., Hooman, K.: Slip-flow in microchannels of non-circular cross sections. Journal of Fluid Engineering 133 (2011). DOI: 10.1115/1.4004591

29. Westerberg, L.G., Lundström, T.S., Höglund, E., Lugt, P.M.: Investigation of grease flow in a rectangular channel including wall slip effects using micro particle image velocimetry. Trib. Trans. 53, 600-609 (2010). DOI: 10.1080/10402001003605566 
Table 1 Rheology data for the used NLGI 00, 1 and 2 greases based on the Herschel-Bulkley rheology model.

\begin{tabular}{llll}
\hline & $\tau_{0}[\mathrm{~Pa}]$ & $K\left[\mathrm{~Pa} \cdot \mathrm{s}^{\mathrm{n}}\right]$ & $n[-]$ \\
\hline NLGI 00 & 1 & 1.85 & 1 \\
NLGI 1 & 182 & 5.1 & 0.75 \\
NLGI 2 & 615 & 10.2 & 0.68 \\
\hline
\end{tabular}

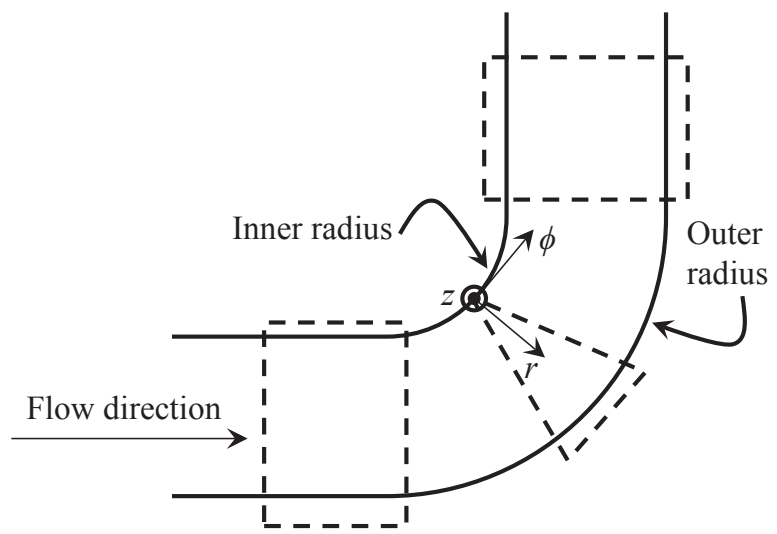

Fig. 1 Schematic drawing of the elbow channel geometries used and the cylindrical coordinate system to describe the flow in the elbow section. The regions marked with dashed lines are the ones where the velocity profiles are measured at the inlet, elbow and outlet respectively.

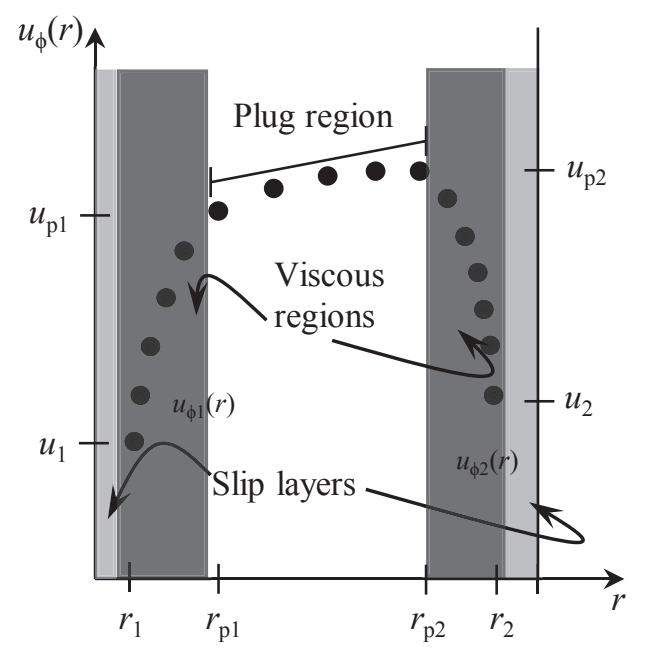

Fig. 2 Schematic view of a typical velocity profile in the channel elbow with a plug region present. Not to scale. The dots represents the measured velocity from the $\mu$ PIV experiments. $r_{1,2}, r_{p 1, p 2}$ and $u_{1,2}, u_{p 1, p 2}$ are the locations and velocity of the first measured velocity value and the location of the yield point respectively in connection to respective channel boundary. 


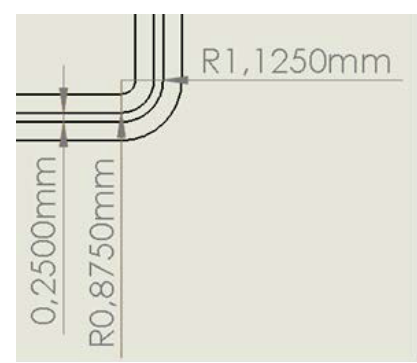

(a)
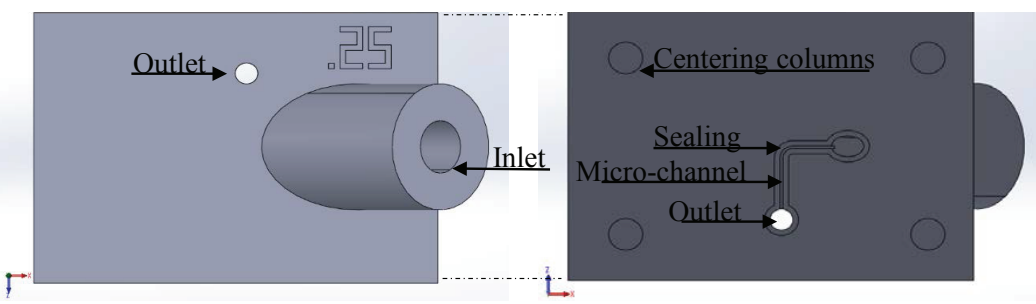

(b)

Fig. 3 Dimensions of the micro-channel (a) and an overview of the channel setup (b). Top view (left) and bottom view (right). 


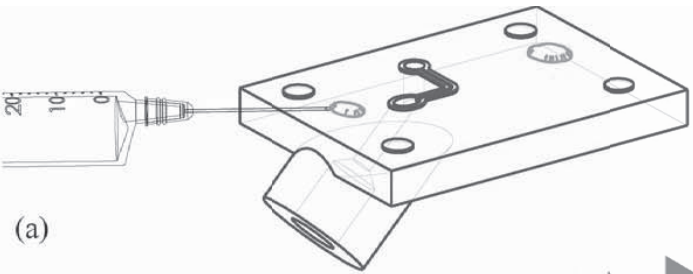

(a)
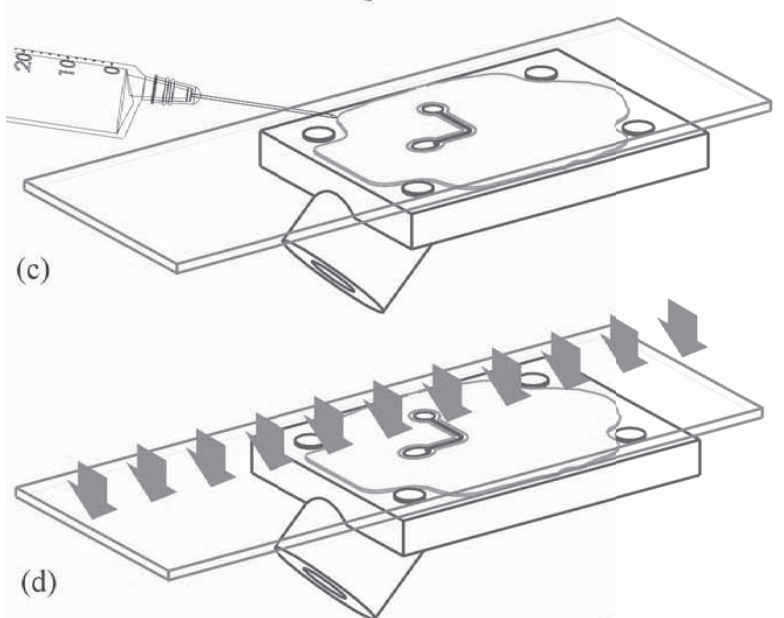

(d)

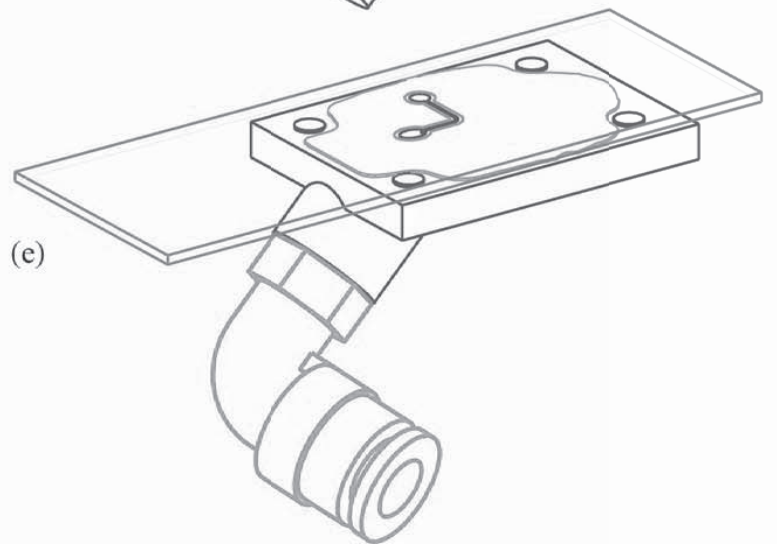

(a) Two UV-curable glue drops are placed on the RPT part and the glass is positioned on top.

(b) UV curing holds the glass.

(c) The gap between the RPT and the glass is filled with UV glue.

(d) The glue is cured

(e) The hydraulic hose is attached using polytetrafluoroethylene.

Fig. 4 Fabrication steps for the micro-channel. 


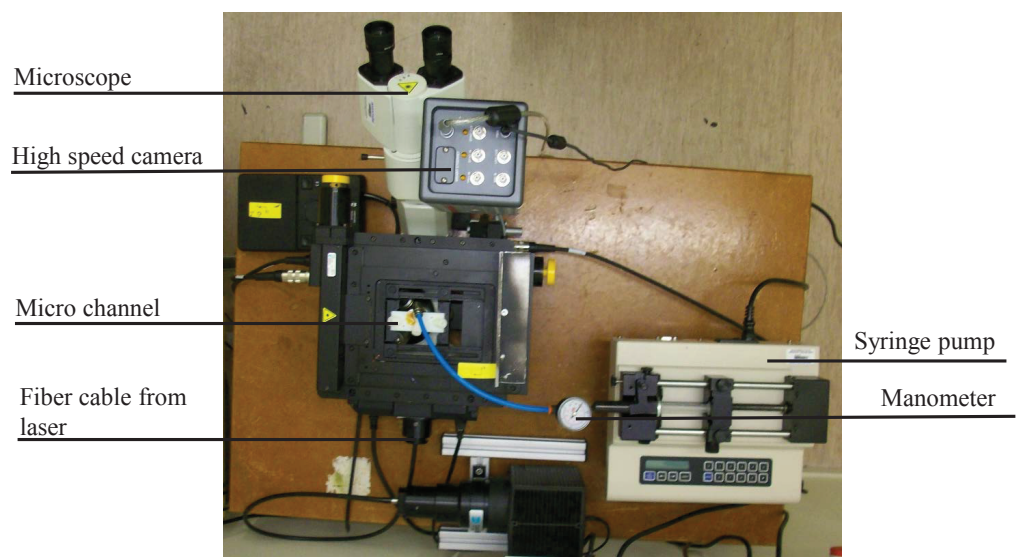

(a)

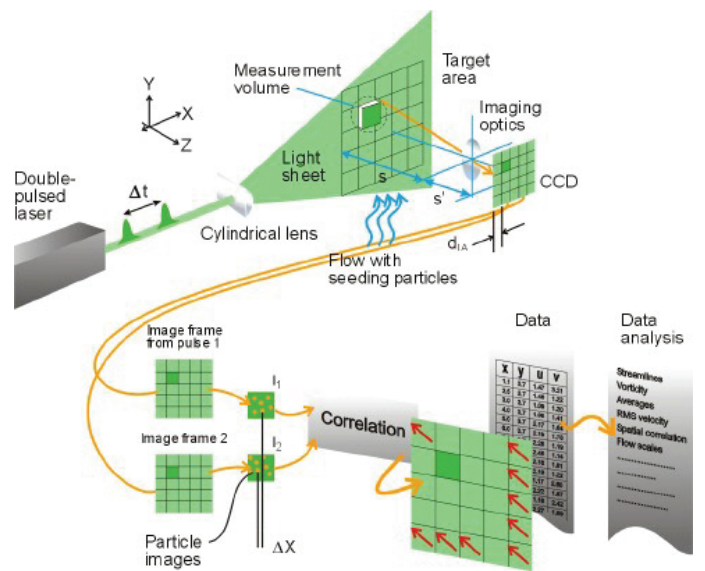

(b)

Fig. 5 Setup of the $\mu$ PIV experiment (a) and the principles of PIV (b). Subfigure (b) is courtesy of Dantec Dynamics A/S. 


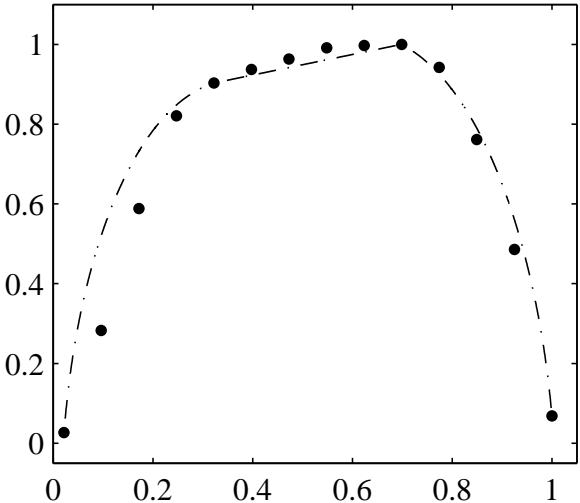

(a) $\alpha=-100$.

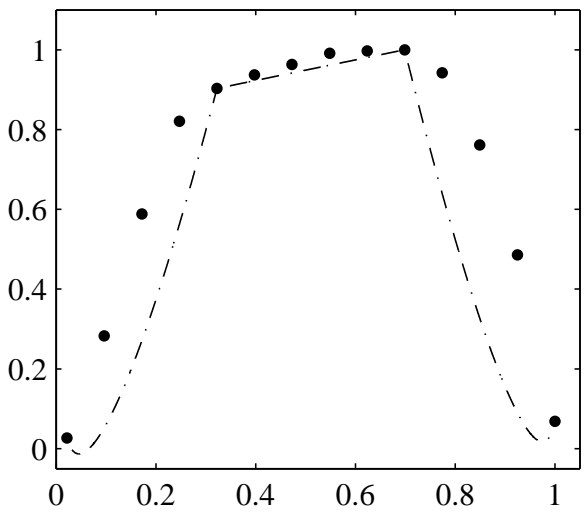

(c) $\alpha=-500$

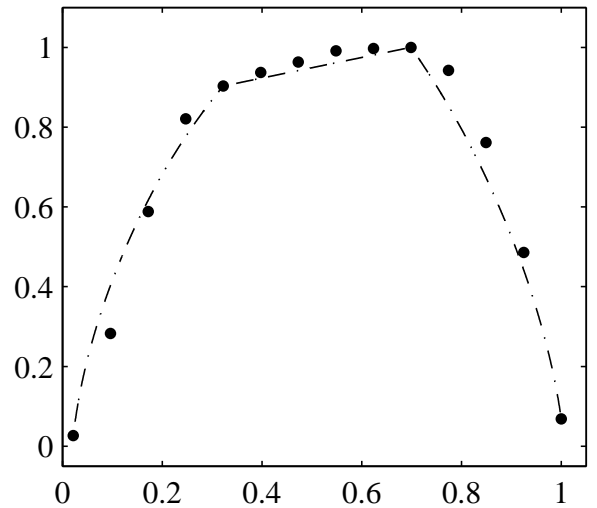

(b) $\alpha=-200$.

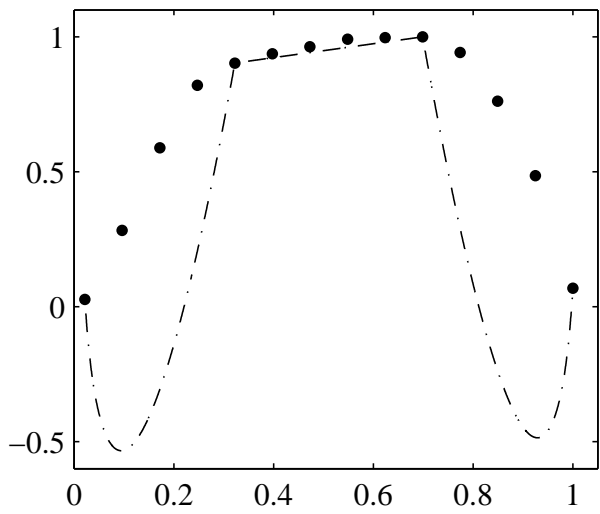

(d) $\alpha=-1000$.

Fig. 6 Dimensionless velocity profiles at the elbow for the NLGI1 grease with a flow rate of $0.02 \mathrm{ml} / \mathrm{min}$. Channel with cross-section of $250 \times 1000 \mu \mathrm{m}$. As characteristic velocity and length scale the maximum velocity and the channel width $(250 \mu \mathrm{m})$ have been used. Dotted: $\mu \mathrm{PIV}$ measurements, dash dotted: analytical model. On the $y$-axis is the dimensionless velocity $\left(u^{*}\left(r^{*}\right)\right)$ and on the $x$-axis is the distance from the inner radius $\left(r^{*}\right) . \alpha$ is the pressure parameter in the analytical model introduced in Eq. (6). Figs. a-d shows the evolution of the analytical solution as the pressure in the $\phi$-direction increases. The values of the rheological parameters in the Herschel-Bulkley rheology model are for these plots: $n=1[-], K=7\left[\mathrm{~Pa} \cdot \mathrm{s}^{\mathrm{n}}\right]$ and $\tau_{0}=160[\mathrm{~Pa}]$. 


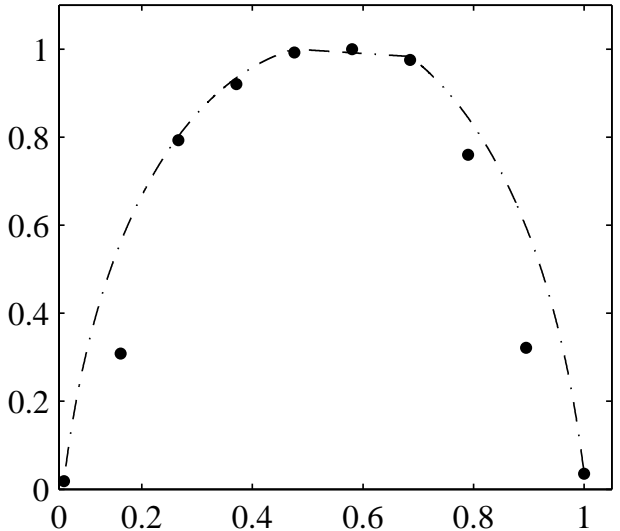

(a) NLGIO0 $2 \cdot 10^{-2} \mathrm{ml} / \mathrm{min}$

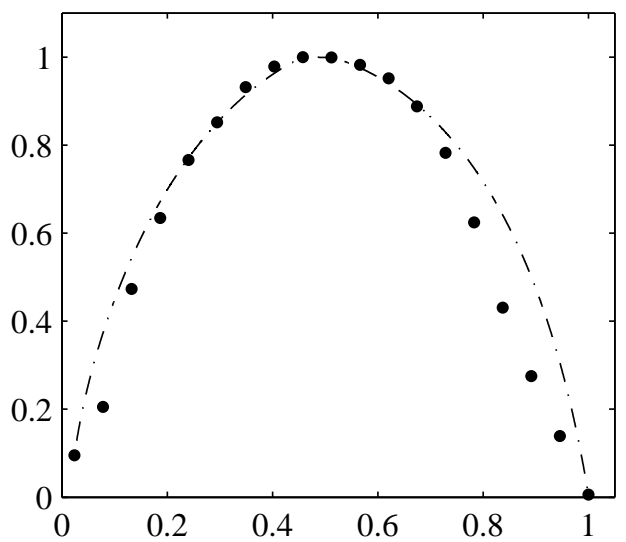

(c) NLGI00 $0.5 \mathrm{ml} / \mathrm{min}$

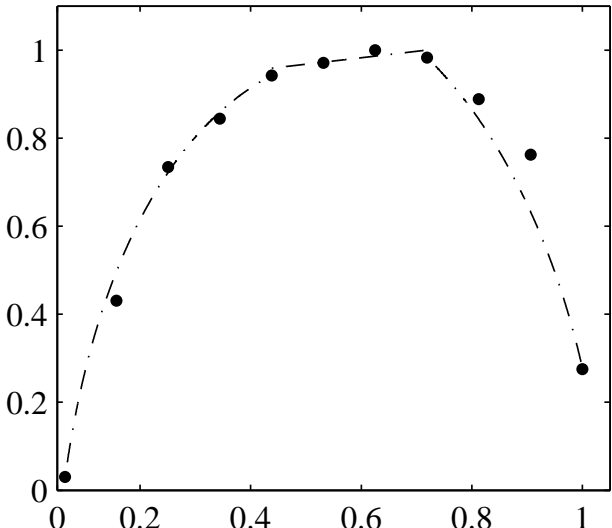

(b) NLGI2 $2 \cdot 10^{-2} \mathrm{ml} / \mathrm{min}$

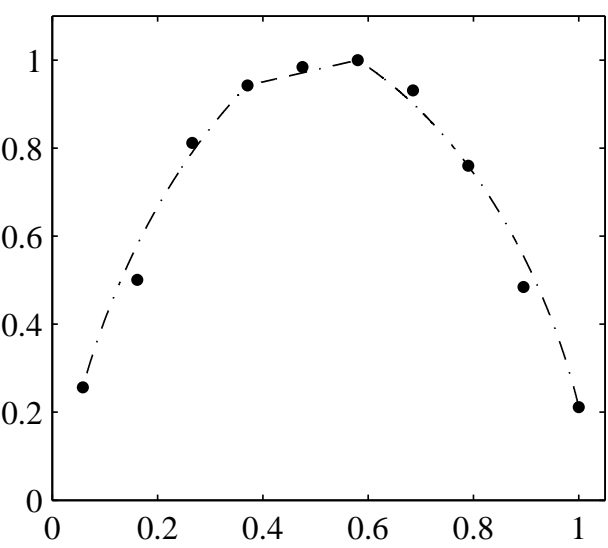

(d) NLGI2 $0.5 \mathrm{ml} / \mathrm{min}$

Fig. 7 Velocity profiles in the channel elbow for the NLGI00- and NLGI2 grease and for two different flow rates. Dotted: $\mu \mathrm{PIV}$ measurements, dash dotted: analytical model. Dimensionless quantities using a characteristic length scale of $250 \mu \mathrm{m}$ (channel width) and a characteristic velocity equal to the maximum velocity. On the $y$-axis is the velocity and on the $x$-axis is the distance from the inner radius. Upper row: flow rate $0.02 \mathrm{ml} / \mathrm{min}$, bottom row: flow rate $0.5 \mathrm{ml} / \mathrm{min}$. 


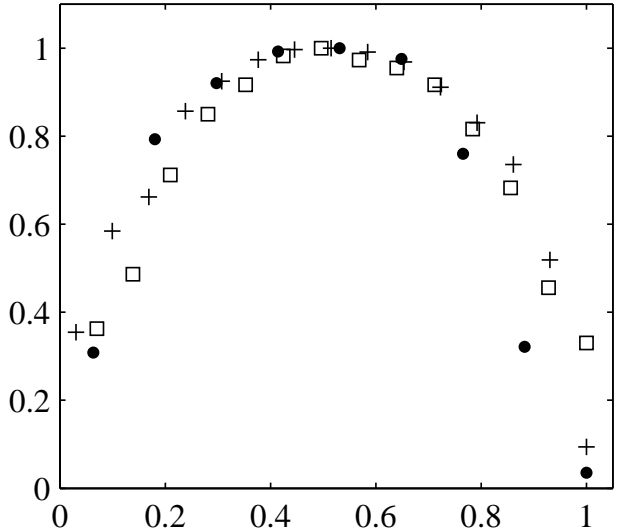

(a) NLGI00 $2 \cdot 10^{-3} \mathrm{ml} / \mathrm{min}$

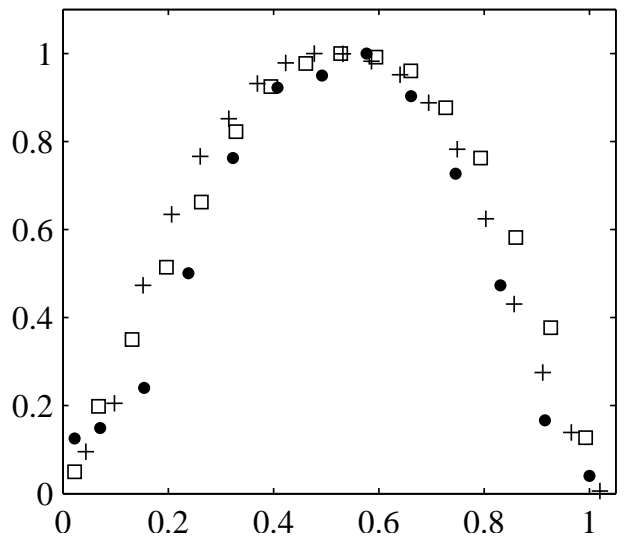

(c) NLGI00 $0.5 \mathrm{ml} / \mathrm{min}$

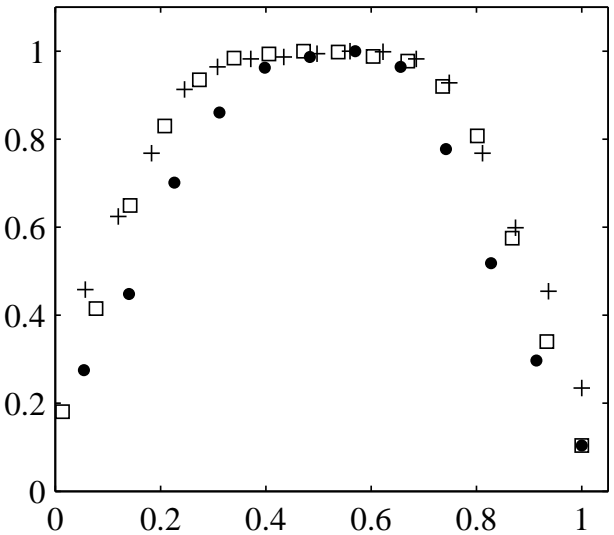

(b) NLGI2 $2 \cdot 10^{-3} \mathrm{ml} / \mathrm{min}$

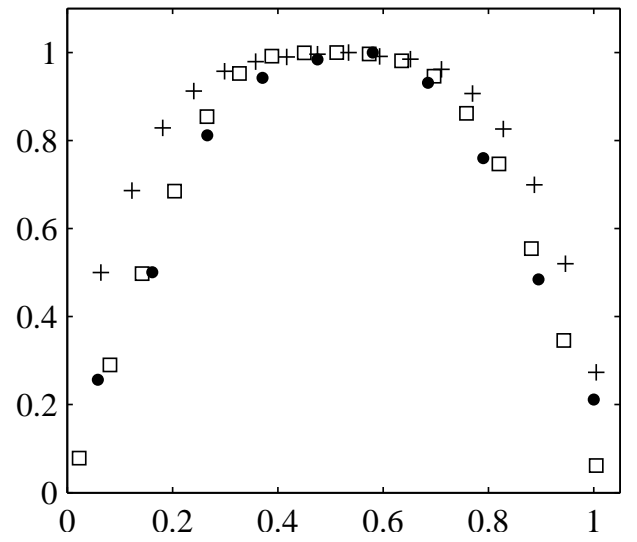

(d) NLGI2 $0.5 \mathrm{ml} / \mathrm{min}$

Fig. 8 Measured velocity profiles at the inlet (square), elbow (dotted), and outlet (cross). Dimensionless quantities using a characteristic length scale of $250 \mu \mathrm{m}$ (channel width) and a characteristic velocity equal to the maximum velocity. On the $y$-axis is the velocity and on the $x$-axis is the distance from the inner radius. Upper row: low flow rate $(0.002 \mathrm{ml} / \mathrm{min})$, bottom row: high flow rate $(0.5 \mathrm{ml} / \mathrm{min})$. 


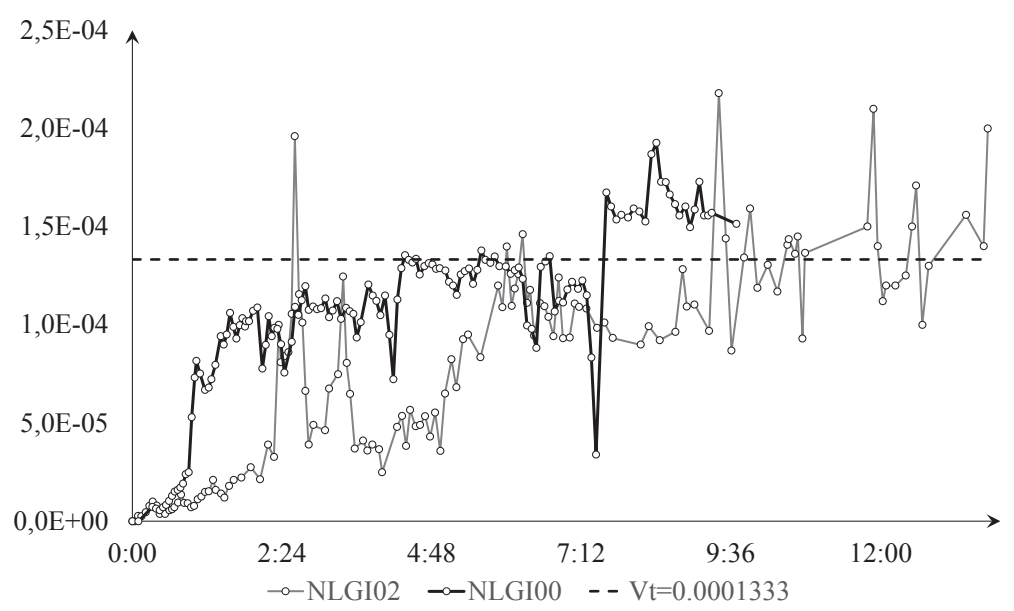

(a) Low flow rate $(0$

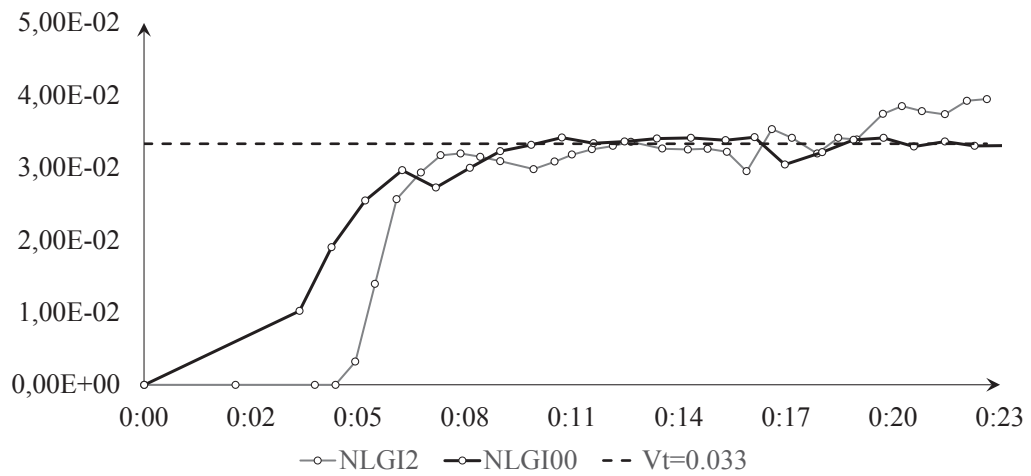

(b) High flow rate

Fig. 9 Speed evolution of the grease flow during the transitory period measured at a control point in the straight section upstream of the elbow (cf. Fig. 1) for the three greases used. On the $y$-axis is the speed in $\mathrm{m} / \mathrm{s}$ and on the $x$-axis the elapsed time (hh:mm). $V_{t}$ is the theoretical speed considering the flow rate and cross section area of the channel assuming a uniform velocity distribution. 